\title{
Research on the Application of Water Resources Rational Allocation of Water Resources in Guizhou Mountainous Area
}

\author{
Min Liu \\ Guiyang University, Guizhou, China 550005
}

\begin{abstract}
In view of the characteristics of the mountain Irrigation District in Guizhou, we combine the characteristics of crop growth and the demand of environmental factors, and the application of the information management system of water resources in Guizhou mountainous areas is carried out. Through monitoring, transmission, diagnosis, decision-making and water dynamic management, according to the crop growing period and other information, the system has achieved accurate irrigation and water resource area reasonable allocation. It has good expansibility, and can meet the individual needs of different farmers according to irrigation scale, cost and crop type, and fully tap the potential of agricultural water saving and efficiency in irrigation district.The System has broad application prospects.
\end{abstract}

Keywords-Guizhou mountainous area; rational allocation of water resources; information management system; application research

\section{INTRODUCTION}

Guizhou mountain areas mainly can can be divided into three categories, sloping hills and plain. Poor soil, poor soil, water resources total quantity is rich but the spatial and temporal distribution is uneven, soil water conservation ability difference, ecological environment is extremely fragile. In addition to take measures to optimize the allocation of water resources is also an important way to improve the allocation and use of water resources and the use of water resources to achieve sustainable use of water resources. Water resources reasonable allocation of information management system is mainly for the current water resources in mountainous areas of our province is extensive management, water resource allocation has not combined with crop growth characteristics and water demand, resulting in waste of water resources and irrigation water use efficiency is low ${ }^{[1]}$. In this paper, the use of computer and network communication technology, developing mountain area irrigation area water resources rational allocation of information management and in Sinan Tang tou modern water conservancy pilot district, formed a group of promotion, you can copy the achievements and experience of modern agriculture development model ${ }^{[1]}$.

\section{OVERVIEW OF INFORMATION MANAGEMENT SYSTEM}

System to cloud computing means, intended to form a suitable for Guizhou mountainous area practicality strong set of irrigation area environment parameters acquisition and monitoring, intelligent irrigation control, integrated management of water and fertilizer, remote wireless video monitoring, pump water energy management, water metering and fee levy elements is one of the water conservancy data information management system, realize the intelligent irrigation, precision fertilization and unmanned management, reduce the management of water resources management and operation cost, improve water resources use efficiency, on behalf of the development direction of modern water conservancy of new technologies, accelerate the agricultural modernization in Henan Province ${ }^{[2]}$.

\section{MAIN FUNCTION MODULE OF SYSTEM}

Human computer interface (UI interface) is implemented by $\mathrm{B} / \mathrm{S}$ architecture, which is convenient for remote client access and centralized management. Other subsystems are composed of $\mathrm{C} / \mathrm{S}$ architecture, through the network connection, each responsible for specific functions. UI interface using Java to achieve a variety of specific application needs and web interface. On the whole we will Web interface system as a system to provide users with specific business implementation. The rest of the system is the background of the system, to provide the functions of the business functions to achieve. Each subsystem is based on the TCP/IP protocol and the data communication is realized. In order to realize the system, we designed and implemented the instruction and data transmission system based on XML, which is used in the network communication.

In the project design, the use of fa? Ade design pattern, the fa? Ade is also known for appearance model, subsystem (or structure and method of all kinds provide a simple and consistent interface and hide the complexity of subsystems, the subsystem easier to use. In this project, the back-end analysis applications require data and application in accordance with the actual situation may be considerable changes. Therefore, it is necessary to application layer shielding subsystem components, reduce client invocation logic, the client application development easier, which focus on user needs logic implementation. In this project, we use the middle control management platform, the system of the complex system to realize the details, which makes the UI component only need to understand the communication protocol of the intermediate control platform. At the same time, this kind of design pattern also provides the convenience for the following distributed deployment, multi service and so on. The UI layer has no need to consider whether it is concurrent, only need to understand the calling efficiency, and the 
optimization of the front and rear platform can be carried out independently by ${ }^{[3]}$.

According to the modular program design idea, system function module mainly irrigation area environment parameter acquisition module, fertilizer integration module, system communication module, query module, statistical report module and system management module analysis etc..

\section{OPTIMAL ALLOCATION MODEL OF SYSTEM}

\section{A. Water Resources Allocation Model}

\section{1) Objective function:}

a) Economic efficiency target: In the model of regional water resources optimal allocation, the economic benefit is one of the main targets, and the indicator is also reflected the social effect. For this project, the economic benefits of the project area is the largest.

$$
f_{1}(x)=\max 1=\max \sum_{k=1}^{K}\left(\sum_{i=1}^{l(k)} \sum_{j=f(j)} F_{k j} x_{i j}^{k} Q_{k j}\right)
$$

$\mathrm{F}_{\mathrm{kj}}$ is unit water output coefficient of $\mathrm{K}$ sub district J,Yuan / square; $\mathrm{X}_{\mathrm{ij}}^{\mathrm{k}}$ is $\mathrm{K}$ District $\mathrm{J}$ department from the water demand in the amount of water required to account for the total amount of water in the $\mathrm{K}$ District of the total amount of water required to configure the proportion of $\mathrm{I} ; \mathrm{Q}_{\mathrm{kj}}$ is water demand in the $\mathrm{j}$ area of K District,Million m3/a.

b) Social efficiency target: From the perspective of social stability and development, the degree of regional water shortage will directly affect social stability and development, therefore, the choice of water shortage as a target of social indicators.

$$
f_{2}(x)=\min 2=\min \sum_{K=1}^{K} \sum_{i=1}^{l(k)}\left(D_{k i}-\sum_{j=1}^{J(k)} x_{i j}^{k} Q_{k j}\right)(2)
$$

$\mathrm{D}_{\mathrm{ki}}$ is $\mathrm{K}$ sub district water supply from I,Million m3/a.

c) Environmental efficiency target: The minimum pollution emission is the environmental objective of water resources optimal allocation.

$$
f_{3}(x)=\min 3=\min \sum_{K=1}^{K} \sum_{j=1}^{J(k)} \sum_{i=1}^{l(k)} a_{k j}\left(1-b_{k}\right) \cdot\left(x_{i j}^{k} Q_{k j}\right)
$$

$a_{k j}$ is Wastewater discharge rate of $\mathrm{J}$ unit in $\mathrm{K}$ sub district,b $\mathrm{k}$ is Wastewater treatment rate in $\mathrm{K}$ sub district.

2) Model solving: We combine the actual situation of engineering and water resources supply and demand balance, to establish the economic and social objective function, in the water and water supply and other constraints to optimize the allocation of water resources. According to the model expression and constraint conditions, the optimal allocation model of water resources is established by using multi-objective programming method, and the optimal allocation scheme of water resources in different level of the project area is obtained by using Studio Visual 2008 software.

\section{B. Stem Irrigation Quota Setting}

Irrigation quota with reference to the "DB52/T" (725-2011), and the relevant results, and according to the irrigation methods, guarantee rate and the actual situation of the local adjustment. Project area in Tongren warm summer weight arid region (zone II), of which $80 \%$ guarantee rate, net of rice irrigation quota $305 \mathrm{~m}^{3} / \mathrm{mu}$, rapeseed $50 \mathrm{~m}^{3} / \mathrm{mu}$, grape $65 \mathrm{~m}^{3} / \mathrm{mu}$, fine fruit (peach, plum, loquat, guava, etc.) $65 \mathrm{~m}^{3} /$ mu, fruit seed seedling breeding $130 \mathrm{~m}^{3} / \mathrm{mu}$, open leaf vegetables $50 \mathrm{~m}^{3} / \mathrm{mu}$, outdoor fruits and vegetables $60 \mathrm{~m}^{3} /$ $\mathrm{mu}$, canopy leaf vegetables $60 \mathrm{~m}^{3} / \mathrm{mu}$, the greenhouse melon and fruit vegetables $70 \mathrm{~m}^{3} / \mathrm{mu}$, the ecological culture of crab $500 \mathrm{~m}^{3} / \mathrm{mu}$.

\section{SYSTEM HARDWARE DESIGN}

The following characteristics of the information management system: (1) to the soil moisture data as the precise irrigation control index. Real-time collecting soil moisture data and the system database design of the data analysis comparison and control irrigation, according to the different growth stage of the crop water demand requirements of precision irrigation; (2) automatic irrigation, saving energy and water. According to the requirement of the preset water demand database, it is necessary to carry out irrigation; (3) the flow, pressure measurement and automatic transmission and distribution of water. Irrigation system is an important network watershed to install the remote meter, pressure gauge, an electromagnetic valve, pressure real-time monitoring, respectively, to control regional irrigation optimal allocation of water resources; (4) and convenient control and management. Control sites can be used for field control, management of room control, remote network remote control, also can use the phone to control. The main hardware design and selection of the main hardware is required for its characteristics.

\section{SYSTEM SOFTWARE DESIGN}

\section{A. Program Design}

After power up the system, enter the initialization state. When the system data is normal, the system can accomplish the following tasks: to obtain the soil moisture, set the temperature and humidity limit and irrigation schedule time, automatic control of irrigation, manual control irrigation.

When the manager sets the control mode for automatic control, the system has the function of timing and adaptive irrigation. If the current time is equal to the planned irrigation time, irrigation is practiced, or else the irrigation is stopped. Program design flow chart is shown in Figure 1.

\section{B. System Application and Effect}

1) Application location: In 2015 June in Sinan Tang tou modern water conservancy pilot District boutique district application area for $15 \mathrm{hm} 2$ plots division rule 37 counties in the District, real-time collecting soil moisture, irrigation pipe network water, and automatic configuration of water resources. Managers set up the soil moisture content range and regional 
irrigation quota, when the system detects that the current soil moisture is lower than the normal growth of crops, the system automatically implemented irrigation; when the system detected the current soil moisture is greater than or equal to the set limit, the system automatically stops irrigation. The system of automatic implementation of total control and quota management of water use principle, metering and progressive increase over quota principle .

2) Application effect: Guizhou mountain area irrigation area water resources reasonable configuration information management the system communication is stable, accurate data acquisition, fast response, managers can through the login password, modify the parameters related to the limit value, trend graph display effect is good, operation interface is beautiful, can meet the production actual requirements, with drought and waterlogging, automatic and scientific configuration of water resources, water high efficiency, saving water and fertilizer advantages. The system can also be applied to the water resources management of the economic crops, such as tobacco, corn and grape.

\section{CONCLUSION}

We have taken the Guizhou mountain area characteristics of fruits and vegetables for example to carry out applications, and completed the design of the three subsystems, multi-objective optimization allocation model is established, completed the system hardware selection and configuration, system software design and development, and in January 2015 in Sinan Tang bea efficient agriculture demonstration base of operation, it indicated that the system has strong adaptability, open, easy expansion, economy and good, can save water resources, to meet the different growth stage of crops suitable for the crop water requirements, improve the quality of crops; no need of electricity wiring, saving labor, energy efficiency, with very good value of application and popularization.

\section{ACKNOWLEDGEMENTS}

This work was financially supported by the project of science and technology of Guizhou province (QKH J Certificate [2013]50nd).

\section{CORRESPONDING AUTHOR}

Liu Min, Instructor, Mainly engaged in computer application and data mining algorithms , E-mail: 253701858@qq.com

\section{REFERENCES}

[1] Zhang He-xi, Yang Jin, Fang Xiao-Yu. Appljcation of Time Series Analysjs in Soil Moisture Forecast [J].Research of Soil and Water Conservation,2008,15(4):82-84.

[2] Mathis W, Larry O, Patricia B, et al. National natural capital accounting with the ecological footprint concept [J]. Ecological Economics, 1999, 29:375-390.

[3] Yang Shu-zhi, Wu Ya. Engineering application of time series analysis[M].Wu Han: Huazhong university of science and technology press,1991.

[4] Wu Xian-ming. Analysis and Application of Time Sequence and System[M].Beijing: Machinery industry press,1988. 


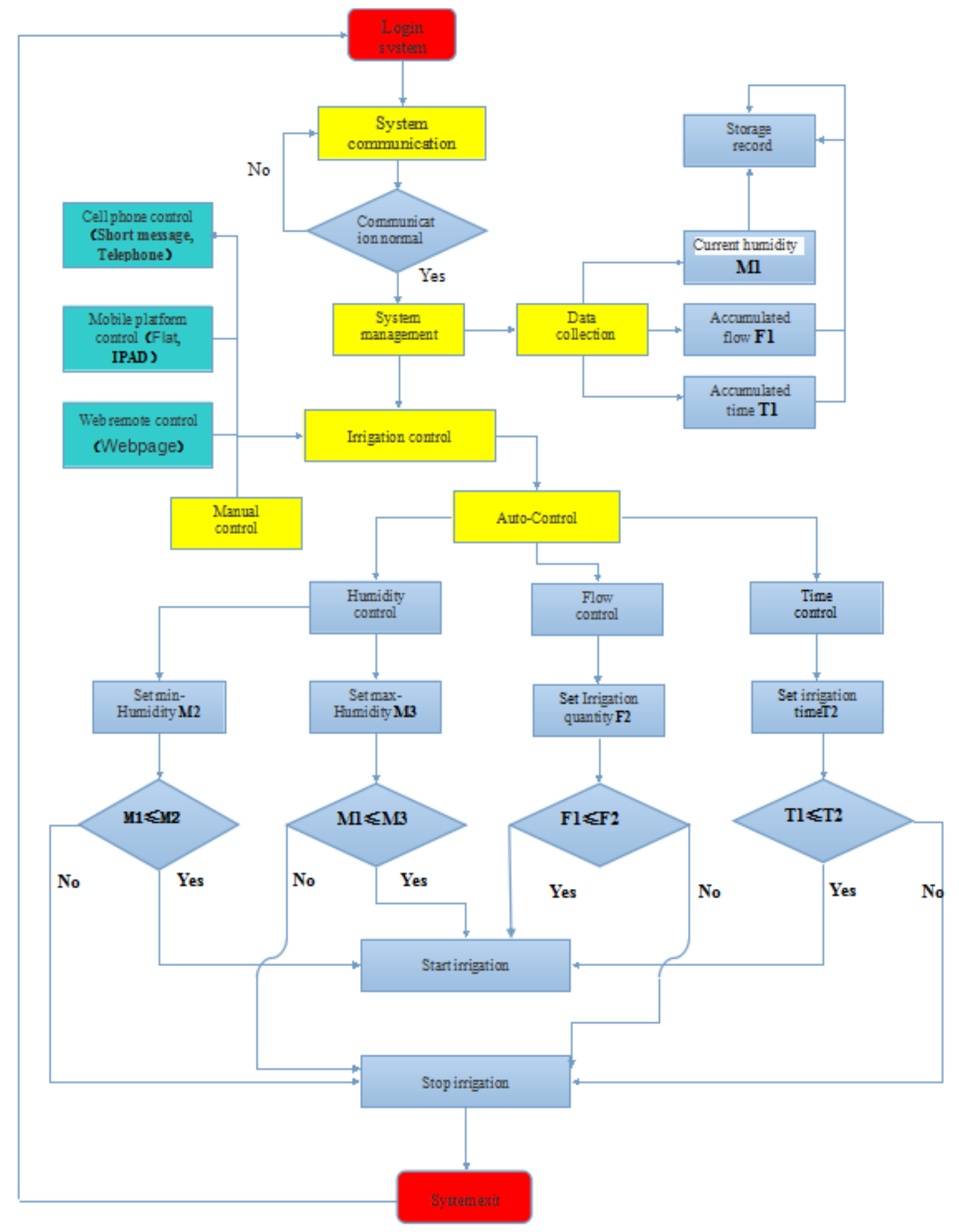

FIGURE I. PROGRAMMING FLOW CHART. 